Such dramatic benefit compelled FDA to approve the drug, says Robert Smith, professor of medicine at the Warren Alpert Medical School of Brown University in Providence, Rhode Island. In an open-label, singlearm study of 48 patients with congenital or acquired generalized lipodystrophy, Myalept decreased hemoglobin A1c levels, a measure of blood glucose, from an average of 8.7 to 6.7. "That's a big effect," says Smith, who chaired FDA's Endocrinologic and Metabolic Drugs Advisory Committee (EMDAC), which voted 11 to 1 in favor of approving the drug to treat generalized lipodystrophy. "A typical diabetes drug in a different setting might decrease HbA1c levels by half that much," he says. Myalept also cut triglycerides by an average of $60 \%$, a more powerful effect than with any other drug, he says.

The regulators also followed the committee's advice in rejecting Myalept for treating partial lipodystrophy. The EMDAC cited a lack of evidence proving the benefits outweighed the risks in this indication. The two most troubling side effects are a potential elevated risk of lymphoma and the risk of developing anti-leptin antibodies. During the trial, three patients developed T-cell lymphoma, although two might have had lymphoma before taking the drug. In theory, the drug could cause lymphoma, as leptin is thought to act on pathways that promote cell growth and inhibit apoptosis through activating the Janus kinase signal transducer and activator of transcription and other intracellular pathways. But individuals with lipodystrophy may be predisposed to lymphoma anyway, so it was difficult to tease out the drug's effects from the disease.

Equally troubling is the risk of inducing anti-leptin antibodies, says Smith. This is particularly the case in people who produce some leptin. "If anti-leptin antibodies develop in these patients, you run the risk of neutralizing leptin they already have and inducing complete lipodystrophy." Three patients out of about 500 in the earlier obesity trials experienced health issues linked to anti-leptin antibodies, but it isn't clear how harmful the antibodies are, says Smith. Nevertheless, because of the risks, Myalept will be available only through the Myalept Risk Evaluation and Mitigation Strategy (REMS) Program.

The path to approval has been a long haul. The initial discovery in 1994 by Jeffrey Friedman at Rockefeller University in New York City, who identified the gene for leptin and the hormone itself, was universally lauded as a breakthrough. The discovery launched a new scientific discipline, the biology of appetite control and obesity research. For the industry, it pointed to a blockbuster weightloss drug. Thousand Oaks, California-based Amgen licensed leptin from Rockefeller for \$20 million and developed metreleptin, a 147 amino acid, nonglycosylated polypeptide with one disulfide bond, which differs from the human leptin sequence by the addition of methionine at the amino terminus.

But preliminary results with the drug on obese patients were disappointing. Obese people, already brimming with leptin, are leptin insensitive. Adding more leptin has no effect. By the end of 1999, Amgen had almost resigned the drug to the junk heap.

In the meantime, despite industry's opprobrium, researchers at the National Institutes of Health (NIH) continued to think of ways to help people with lipodystrophies. In 1997, Simeon Taylor and Elif Oral at NIH's Institute of Diabetes and Digestive and Kidney Diseases were recruiting lipodystrophy patients to take part in a clinical trial of Rezulin (troglitazone), a diabetes drug since discontinued. In some patients who were excluded owing to poor liver function, Oral measured leptin levels to figure out another way to help them. "At the time measuring leptin was all the rage, it's what you did," she adds. The sickest patients had very low leptin levels. The investigators wondered whether leptin supplements might alleviate metabolic complications. Oral approached Amgen without success. At the time, Amgen's obesity studies were ongoing and the company was still hoping for a blockbuster, she says.

Then research fingering leptin to treat lipodystrophy started to heat up. O'Rahilly and his colleagues at Cambridge published the first study identifying a rare group of children with a congenital form of leptin deficiency, who were morbidly obese (Nature 387, 903-908, 1997). Moreover, his group showed in 1999 that daily injection of recombinant leptin therapy (supplied by Amgen on a compassionate-use basis) helped dramatically (N. Engl. J. Med. 341, 879-884, 1999).

About the same time, Michael Brown and Joseph Goldstein, both Nobel laureates at UT Southwestern, were studying how the body regulates cholesterol. Led by Iichiro Shimomura, they had created a transgenic mouse that overexpressed sterol regulatory element binding protein 1 (SREBP-1) and unwittingly developed a model of congenital lipodystrophy (Genes Dev. 12, 3182$3194,1998)$. Giving leptin to the transgenic SREBP-1 mice also improved metabolic biomarkers.

All the while, Oral kept bugging Amgen. "When you are dealing with a really sick patient and you don't know what to do, you
IN brief

\section{Epilepsy neurodevice approved}

A new generation of surgically implanted neuromodulation devices is making regulatory headway with one recent approval from the US Food and Drug Administration (FDA) and a positive recommendation. In November, the FDA granted premarket approval to Mountain View, California-based NeuroPace's responsive neurostimulation system (RNS), a pulse generator implanted in the brain that detects and disrupts epilepsy seizures by delivering normalizing electrical neurostimulation. The system is approved for individuals for whom two antiepileptic drugs fail to control seizures More recently, in February, the FDA's Anesthesiology and Respiratory Therapy Devices Panel recommended approval of the Inspire II Upper Airway Stimulator, developed by Inspire Medical Systems, of Maple Grove, Minnesota, to treat obstructive sleep apnea in individuals who have failed continuous positive-airway pressure treatment. The device implanted under the chest skin senses breathing patterns, delivering mild stimulation to the airway muscles and the hypoglossal nerve, which runs beneath the base of the tongue, to keep the airways open during sleep. These 'closedloop' systems are a dramatic departure from previous neurostimulation devices as they rely on sensors to anticipate neurostimulation needs. Joseph S. Neimat, an associate professor of neurological surgery at Vanderbilt University in Nashville, Tennessee, says, "What we've done in the past is a one-way stimulation model.... You pretty much put the stimulator in, you turn it on, you let it fire X number of times in a particular pattern." Instead, the closed-loop stimulators "listen," he says. As knowledge of brain function continues to grow, "There are going to be more proposals in front of the FDA for devices like this," Neimat predicts. Alli Proffitt

\section{IN their words}
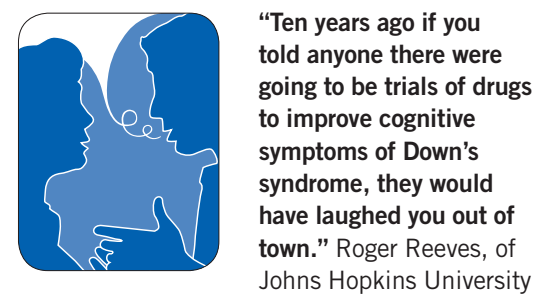

in Baltimore, on Roche's partnering with Balance Therapeutics of San Bruno, California, to test GABA-blocking drugs to improve memory. Similar drugs improved surrogates of memory in a Down's syndrome mouse model. (Bloomberg Sustainability, 3 March 2014) 